\title{
ERRATUM
}

\section{To the article}

\section{«Heterodimer HLA-DM Fused with Constant Fragment} of the Heavy Chain of the Human Immunoglobulin Accelerates Influenza Hemagglutinin $\mathrm{HA}_{306-318}$ Loading to HLA-DR1»

by A. E. Mamedov, N. A. Ponomarenko, A. A. Belogurov Jr, and A. G. Gabibov, Vol. 161, No. 1, pp. 92-95, May, 2016

The text of the article should be

\section{Heterodimer HLA-DM Fused with Constant Fragment of the Heavy Chain of the Human Immunoglobulin Accelerates Influenza Hemagglutinin Peptide $\mathrm{HA}_{306-318}$ Loading to HLA-DR1}

\section{A. E. Mamedov, N. A. Ponomarenko, A. A. Belogurov Jr, and A. G. Gabibov}

Translated from Byulleten'Eksperimental'noi Biologii i Meditsiny, Vol. 161, No. 1, pp. 101-105, January, 2016 Original article submitted April 26, 2015

\begin{abstract}
Major histocompatibility complex class II (MHC II) plays an important role not only in the adaptive immune responses to foreign pathogens, but also in the development of some autoimmune diseases. Non-classical MHC, HLA-DM is directly involved in MHC II loading with the peptide. To study this process, we obtained recombinant proteins HLA-DR1 and HLADM. $\alpha / \beta$-Chains of DR1 heterodimer contained C-terminal leucine domains of the fos and jun factors, respectively. Each DM chain contained constant fragment of human antibody heavy chain fused via a long linker domain. In addition, DM $\alpha$-chain carried N165D substitution suppressing potential glycosylation at this site. We observed significant acceleration of influenza hemagglutinin peptide $\mathrm{HA}_{306-318}$ loading to DR1 in the presence of DM, which indicates functionality of recombinant DR1-DM protein couple. Our results can be used to study the
\end{abstract}

M. V. Shemyakin and Yu. A. Ovchinnikov Institute of Bioorganic Chemistry, Moscow, Russia.Address for correspondence: alexey.belogurov. jr@gmail.ru. A. A. Belogurov Jr 
presentation of other viral and self-antigens and can become the basis for the development of new drug modeling.

Key Words: major histocompatibility complex class II; HLA-DR1; HLA-DM; hemagglutinin peptide $H A$

Major histocompatibility complex class II (MHC II), a heterodimeric transmembrane glycoprotein with a molecular weight of $50 \mathrm{kDa}$, plays a central role in the adaptive immune response to foreign pathogens. It is expressed by "professional" antigen-presenting cells such as dendritic cells, macrophages, and B cells. After loading, the MHC-peptide complex is transported to the cell surface, where it interacts with T-cell receptors on the surface of $\mathrm{CD}^{+} \mathrm{T}$ cells. Newly synthesized $\alpha$-and $\beta$-subunits of MHC II are translocated into the endoplasmic reticulum, where they bind to the trimeric chaperone protein, MHC II-associated invariant chain [2]. In the absence of peptide, MHC II aggregates [4], i.e. invariant chain initially protects the hydrophobic peptide binding site [8]. Then the complex enters the endosomal compartment [10]. The invariant chain outside the sites involved in trimer formation and $\mathrm{MHC}$ II association is unstructured; it is highly susceptible to proteolysis [7] and can be rapidly degraded by endosomal proteases, e.g. cathepsins [6]. The larger fragment of the invariant chain is eliminated, except a short CLIP peptide that remain bound in the peptidebinding groove of MHC II [7,5].

The peptides assigned for loading on MHC II are formed from host or foreign proteins entering the endosome via pinocytosis, receptor-mediated endocytosis, or autophagy. Irrespective of the origin of the target peptide, CLIP peptide should be removed from MHC II before loading. This process is provided by HLA-DM (DM), non-classical MHC II. The term "non-classical" means the absence of polymorphism and inability to bind peptides in contrast to classical human MHC II: HLA-DR, HLA-DP and HLA-DQ. DM has catalytic activity that promotes both peptide binding and release $[11,14]$, i.e. acts as an enzyme-like catalyst $[12,13]$. DM is needed for efficient peptideloading; in the absence of DM, many MHC II on the cell surface carry CLIP instead of antigenic determinants [3]. It should be noted that despite extensive studies, the mechanism of DM action remains unclear.

Our aim was creation of genetic constructs and expression of functional protein pair HLA-DR1 and HLADM in preparative amounts for more detailed study of DM-dependent presentation of autoantigens on MHC II.

\section{MATERIALS AND METHODS}

In our laboratory, we have previously obtained genetic constructs pMT-V5/His_Fos and pRmHa_Jun encoding leucine motifs of fos transcription factor in case of $\alpha$-chain and jun factor in case of $\beta$-chain to enhance expression and improve stability of the resulting DR-complex for expression in insect cells. The variable and constant domains of both HLA-DR1 chains were amplified by PCR using genomic DNA of human HeLa cell line as the template. The resulting PCR products were ligated into pMT-V5/His Fos and pRmHa Jun constructs at KpnI/AgeI and BamHI/ SalI restriction sites for $\alpha$ - and $\beta$-chain, respectively. For proteins expression in suspension human cell culture HEK, genetic constructs pFUSE_Fc and pFUSE $1 \mathrm{hFc}$ encoding constant fragments of human immunoglobulin heavy chain (Fc) with standard and extended linker (hinge) domain, respectively, were also previously obtained in our laboratory. The variable and constant domains of HLA-DM $\alpha$ - and $\beta$-chains were amplified by PCR using genomic DNA of human HeLa cell line as the template. The resulting PCR products were ligated into pFUSE_Fc and pFUSE_lhFc genetic constructs at EcoRI and NcoI restriction sites. Based on pFUSE DM $\alpha$ Fc, pFUSE $\mathrm{DM} \beta \_F \mathrm{~F}, \mathrm{pFUSE} D \mathrm{DM} \alpha \_\mathrm{hFc}$ and $\mathrm{pFUSE} \overline{\mathrm{E}} \mathrm{DM} \beta \_\mathrm{lhFc}$ and using overlapping PCR technique, we created genetic constructs with point substitutions N165D for $\alpha$-chain and N192D for $\beta$-chain that are believed to be potential glycosylation sites.

Drosophila melanogaster S2 cells were transfected by lipofection technique with pMT-V5/His DRa_Fos and pRmHa_DRb_Jun plasmids as well as a plasmid encoding blasticidin-resistance gene followed by blasticidin selection of stable culture. HLA-DR1 expression in the resulting cell line was performed in serum-free medium Sf9, containing $1 \mathrm{mM} \mathrm{Cu}^{2+}$ over 9 days. The resultant culture medium was 10 -fold concentrated, dialyzed against PBS, applied onto an affinity resin (L243 monoclonal antibodies to HLA-DR complex immobilized on CNBr-activated sepharose), and eluted with $50 \mathrm{mM}$ glycine buffer ( $\mathrm{pH}$ 11.5); the collected fractions were neutralized with $1 \mathrm{M}$ tris- $\mathrm{HCl}$ ( $\mathrm{pH} 8.0)$.

Suspension HEK293F cells were transfected with pFUSE DM $\alpha$ N165D $1 \mathrm{hFc}$ and pFUSE DM $\beta$ lhFc plasmids using lipofection technique. HLA-DM expression was performed in serum-free FreeStyle medium over 9 days. The resultant culture medium was loaded onto Protein $\mathrm{G}$ and eluted with $50 \mathrm{mM}$ glycine buffer ( $\mathrm{pH}$ 2.5); collected fractions were neutralized with $1 \mathrm{M}$ tris- $\mathrm{HCl}(\mathrm{pH} 8.0)$. 


\section{$a$}

HLA-DM chains

$\begin{array}{lll} & \beta & \\ \text { wt } & \text { wt } & \text { DM1 } \\ \text { wt } & \text { N192D } & \text { DM2 } \\ \text { N165D } & \text { wt } & \text { DM3 } \\ \text { N165D } & \text { N192D } & \text { DM4 }\end{array}$

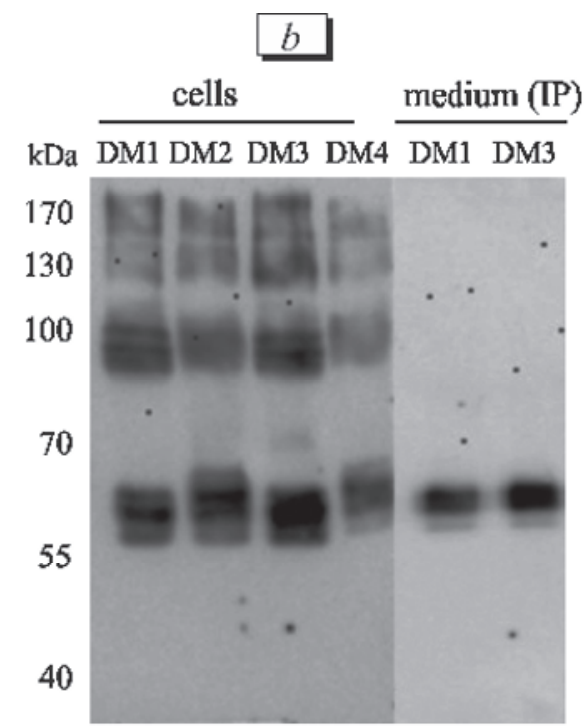

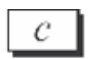

ELISA signal

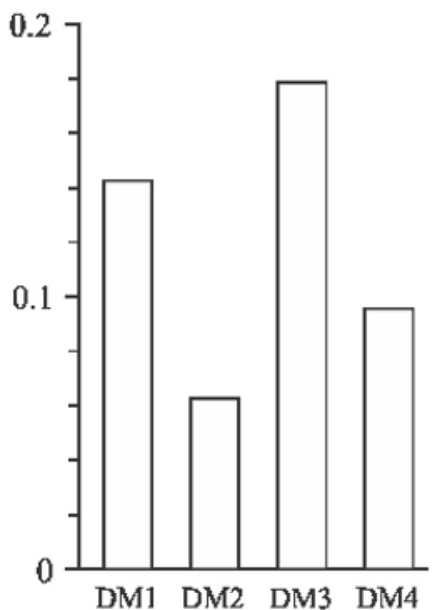

d

ELISA signal

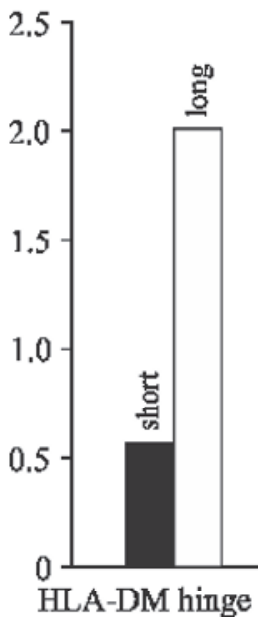

Fig. 1. Analyzed combination of HLA-DM chains (a), Western blot of transient transfection of DM $\alpha / \beta$-chains combinations $(b)$, ELISA expression of DM $\alpha / \beta$-chains combinations in medium $(c)$ and DM3 with standard and extended hinge $(d)$. Immobilized antibodies are monoclonal antibodies to human antibodies, secondary antibodies are horseradish peroxidase conjugated monoclonal antibodies to human Fc-fragment. IP: immunoprecipitation.

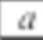

M

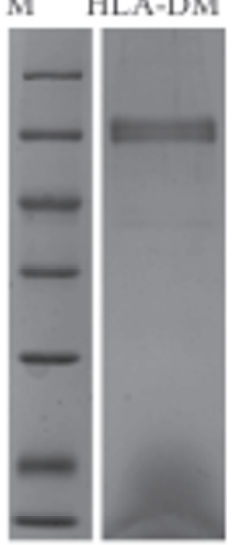

Fig. 2. SDS-PAGE of purified HLA-DM (a), SDS-PAGE and Western blotting of purified HLA-DR1 $(b)$, ELISA of loading reactions of $\mathrm{HA}_{306-318}$ peptide (PKYVKQNTLKLAT; $1.5 \mu \mathrm{M}$ ) onto HLA-DR1 $(0.3 \mu \mathrm{M})$ with and without HLA-DM $(0.3 \mu \mathrm{M})$ (c), kinetics of HA peptide loading reaction on HLA-DR1 over $5 \mathrm{~h}$ with and without HLA-DM (d). Immobilized antibodies are specific monoclonal antibodies L243 to HLA-DR complex, visualization: streptavidin conjugated with horseradish peroxidase. Arrows show bands corresponding to $\alpha$ - and $\beta$-chains.

FT - flowthrough.

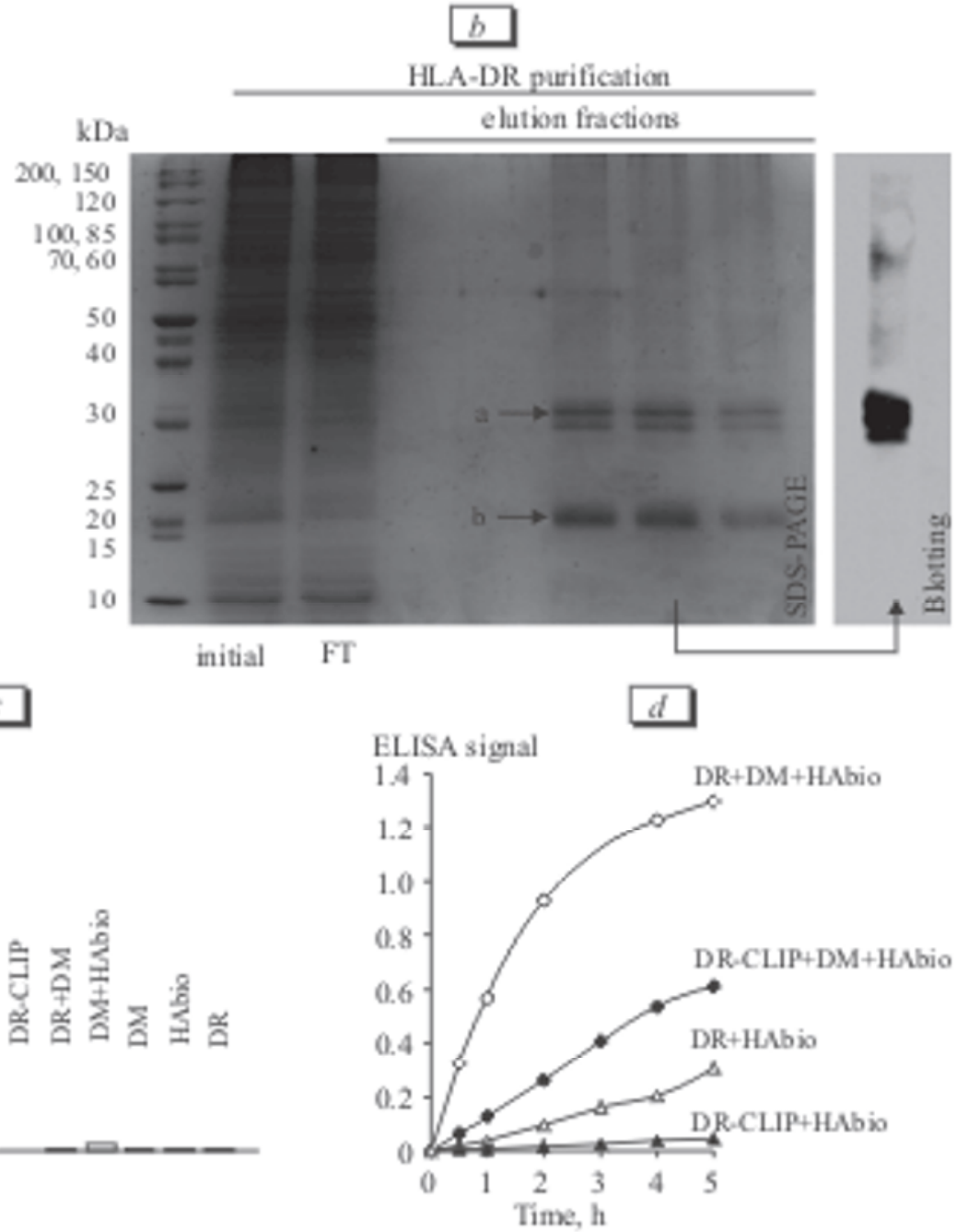


ELISA and Western immunoblotting were performed by standard protocols. For immunoblotting, immunoprecipitation with monoclonal antibodies to the FLAG-epitope immobilized to agarose was carried out.

\section{RESULTS}

Despite large body of evidence about presentation of exogenous and endogenous antigens by MHC II, the mechanism of this process is poorly understood. Elucidation of the process of activation of antigen presentation is very important for understanding of the mechanisms of autoimmune processes and destruction of cells damaged by an external pathogen. In autoimmune pathologies, a number of autoantigen peptides and the corresponding MHC II were identified, for example, myelin basic protein $\mathrm{MBP}_{85-99}$ - HLA-DR2b (DRA, DRB1 1501 allele) complex in multiple sclerosis [15], and collagen II peptide $\mathrm{CII}_{263-270}$ - HLA-DR1 (DRA, DRB1 0101 allele) and HLA-DR4 (DRA, DRB1 0401 allele) complexes in rheumatoid arthritis [9].

In this study, we choose HLA-DR1 allele as the most common in adaptive immunity and the least studied in autoimmune pathologies. The first task was the expression and purification of HLA-DR1 and HLADM proteins and then we tested the hypothesis of acceleration of loading of the chosen HLA-DR allele by HLA-DM. To test this hypothesis and to confirm functional expression of HLA-DR1 and HLA-DM couple, we chose classical influenza virus hemagglutinin peptide $\mathrm{HA}_{306-318}[1]$.

To solve the first task, we fused HLA-DR and HLA-DM with a fragment of human immunoglobulin constant heavy chain $(\mathrm{Fc})$. This modification improves complex stability due to dimerization of $\mathrm{Fc}$, enhances expression, and ensures effective one-step affinity isolation on Protein G. However, in case of HLA-DR, this expression and isolation system was less effective than one with constructs containing fos and jun produced by expression in Drosophila melanogaster S2 cells.

In case of HLA-DM, there was a possibility of protein glycosylation in HEK cells; therefore, we decided to introduce two substitutions in potential glycosylation sites N165D on the $\alpha$-chain and N92D on $\beta$-chain [1] and create four combinations: DM1, DM2, DM3, and DM4 (Fig. 1, a). Transient transfection with the obtained genetic constructs was performed to identify the optimal DM combination. The highest expression level was obtained for DM3 that consisted of $\mathrm{DM} \alpha \mathrm{N} 165 \mathrm{D}$ and wild type $\mathrm{DM} \beta$ (Fig. $1, b, c$ ) This variant was chosen for further studies. At the next stage, we performed transient transfection of the chosen DM3 with standard and extended hinge. According to ELISA data (Fig. 1, $d$ ), the effect of extended hinge that presumably improves DM complex folding 4-fold exceeded the effect of standard hinge.

Finally, DM $\alpha$ N165D $\mathrm{lhFc}$ and $\mathrm{DM} \beta \mathrm{lhFc}$ were selected for HLA-DM. The yield of HLA-DM was $24 \mathrm{mg}$ from 1 liter medium, purity $\geq 98 \%$ (Fig. 2, a). HLA-DR with fos and jun was obtained by expression in Drosophila melanogaster S2 cells; the yield was $2 \mathrm{mg}$ from 1 liter medium, purity $\geq 95 \%$ (Fig. 2, $b)$. At the last stage we analyzed activation of viral peptide presentation by HLA-DM complex. Loading of HA peptide onto HLA-DR1 after $1 \mathrm{~h}$ incubation was detected only in the presence of HLA-DM in the reaction mixture (Fig. 2, c). Detailed analysis showed that HLA-DR 10-fold accelerated the loading process in comparison with the non-catalyzed reaction (Fig. 2, $d$ ).

It is known that apart from classical protective functions MHC II is involved in the development of autoimmune diseases such as diabetes type 1, rheumatoid arthritis, and multiple sclerosis. Autoimmune pathologies attract much interest from the viewpoint of modern experimental molecular immunology, because the mechanisms underlying the development of these diseases are not yet completely understood. Our experiments showed that HLA-DM assists presentation of the viral peptide by HLA-DR complex. The designed genetic constructs and chosen conditions for the expression of complex components in insect and mammalian cells will be useful for studying the mechanism of autoantigen fragments presentation by MHC II HLA-DR2b in multiple sclerosis.

The study was supported by Ministry of Education and Science of the Russian Federation (RFMEFI61314X0008).

\section{REFERENCES}

1. A. K. Anders, M. J. Call, M. S. Schulze, K. D. Fowler, D. A. Schubert, N. P. Seth, E. J. Sundberg, and K. W. Wucherpfennig, HLA-DM captures partially empty HLA-DR molecules for catalyzed removal of peptide. Nat Immunol., 12, No. 1, 54-61 (2011).

2. C. Cresswell, Assembly, transport, and function of MHC class II molecules. Annu. Rev. Immunol., 12, 259-293 (1994).

3. L. K. Denzin and C. Cresswell, HLA-DM induces CLIP dissociation from MHC class II alpha beta dimers and facilitates peptide loading. Cell, 82, No. 1, 155-165 (1995).

4. R. N. Germain and A. G. Rinker Jr, Peptide binding inhibits protein aggregation of invariant-chain free class II dimers and promotes surface expression of occupied molecules. Nature, 363, 725-728 (1993).

5. P. Ghosh, M. Amaya, E. Mellins, and D. Wiley, The structure of an intermediate in class II MHC maturation: CLIP bound to HLA-DR3. Nature, 378, 457-462 (1995).

6. C. S. Hsieh, P. deRoos, K. Honey, C. Beers, A. Y. Rudensky, A role for cathepsin $\mathrm{L}$ and cathepsin $\mathrm{S}$ in peptide generation for MHC class II presentation. J. Immunol., 168, No. 6, 2618-2625 (2002). 
7. A. Jasanoff, S. J. Park, and D. Wiley, Direct observation of disordered regions in the major histocompatibility complex class II-associated invariant chain. Proc. Natl Acad. Sci. USA, 92, No. 21, 9900-9904 (1995).

8. P. A. Roche, C. Cresswell, Invariant chain association with HLA-DR molecules inhibits immunogenic peptide binding. Nature, 345, 615-618 (1990).

9. E. F. Rosloniec, K. B. Whittington, D. M. Zaller, and A. H. Kang, HLA-DR1 (DRB1*0101) and DR4 (DRB1*0401) use the same anchor residues forbinding an immunodominant peptide derived from human type II collagen. J. Immunol., 168, No. 1, 253-259 (2002).

10. L. Santambrogio, I. Potolicchio, S. P. Fessler, S. H. Wong, G. Raposo, and J. L. Strominger, Involvement of caspasecleaved and intact adaptor protein 1 complex in endosomal remodeling in maturing dendritic cells. Nat. Immunol., 6, No. 10, 1020-1028 (2005).
11. M. A. Sherman, D. A. Weber, and P. E. Jensen, DM enhances peptide binding to class II MHC by release of invariant chainderivedpeptide. Immunity, 3, No. 2, 197-205 (1995).

12. V. S. Sloan, P. Cameron, G. Porter, M. Gammon, M. Amaya, E. Mellins, and D. M. Zaller, Mediation by HLA-DM of dissociation of peptides from HLA-DR. Nature, 375, 802-806 (1995).

13. A. B. Vogt, H. Kropshofer, G. Moldenhauer, and G. J. Hammerling, Kinetic analysis of peptide loading onto HLA-DR molecules mediated by HLA-DM. Proc. Natl Acad. Sci. USA, 93, No. 18, 9724-9729 (1996).

14. D. A. Weber, B. D. Evavold, and P. E. Jensen, Enhanced dissociation of HLA-DR-bound peptides in the presence of HLADM. Science, 274, 618-620 (1996).

15. K. W. Wucherpfennig, A. Sette, S. Southwood, C. Oseroff, M. Matsui, J. L. Strominger, and D. A. Hafler, Structural requirements for binding of an immunodominant myelin basic protein peptide to DR2 isotypes and for its recognition by human T cell clones. J. Exp. Med., 179, No. 1, 279-290 (1994). 